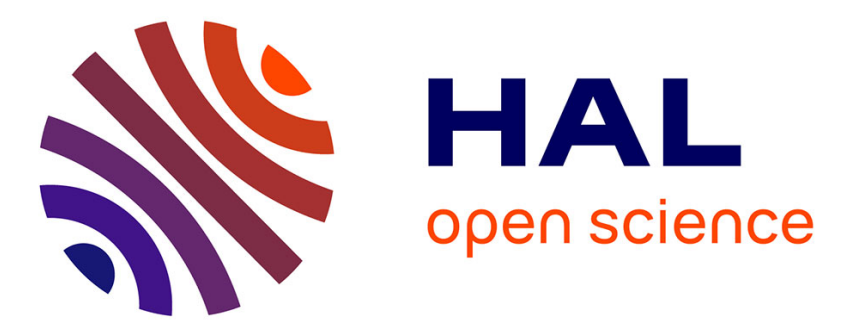

\title{
The Dynamics of Pheromone Gland Synthesis and Release: a Paradigm Shift for Understanding Sex Pheromone Quantity in Female Moths
}

\author{
Stephen P Foster, Karin G Anderson, Jérome Casas
}

\section{- To cite this version:}

Stephen P Foster, Karin G Anderson, Jérome Casas. The Dynamics of Pheromone Gland Synthesis and Release: a Paradigm Shift for Understanding Sex Pheromone Quantity in Female Moths. Journal of Chemical Ecology, 2018, 44 (6), pp.525-533. 10.1007/s10886-018-0963-z . hal-02573239

\author{
HAL Id: hal-02573239 \\ https://hal.science/hal-02573239
}

Submitted on 14 May 2020

HAL is a multi-disciplinary open access archive for the deposit and dissemination of scientific research documents, whether they are published or not. The documents may come from teaching and research institutions in France or abroad, or from public or private research centers.
L'archive ouverte pluridisciplinaire HAL, est destinée au dépôt et à la diffusion de documents scientifiques de niveau recherche, publiés ou non, émanant des établissements d'enseignement et de recherche français ou étrangers, des laboratoires publics ou privés. 


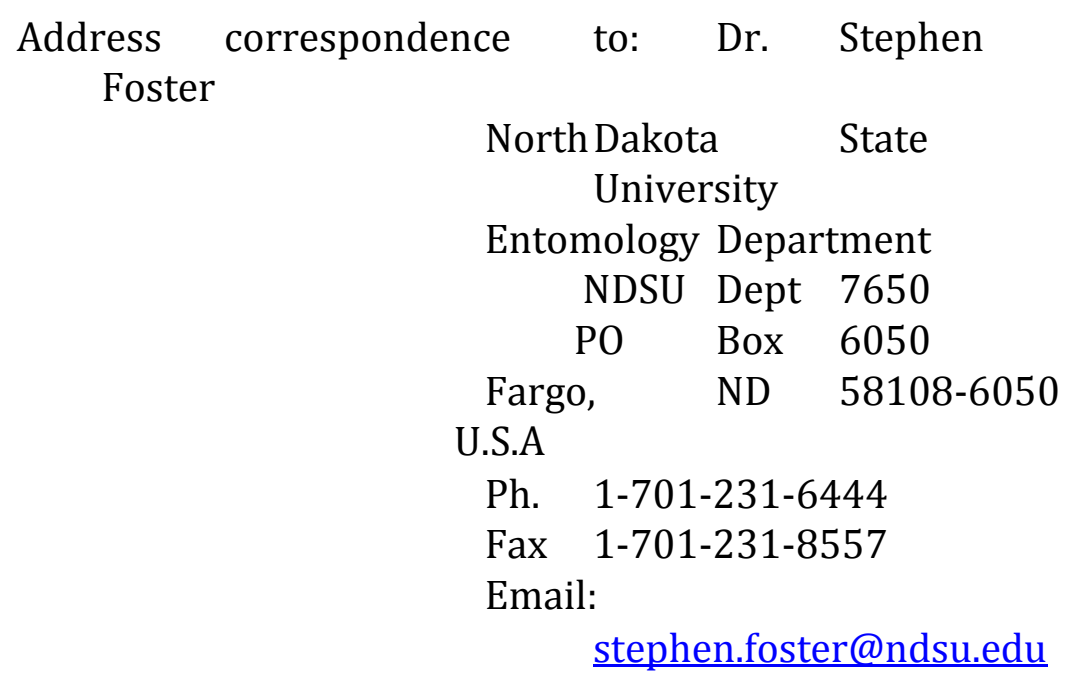

\title{
The dynamics of pheromone gland synthesis and release: a paradigm shift for understanding sex pheromone quantity in female moths
}

\author{
Stephen P. Foster ${ }^{1}$, Karin G. Anderson ${ }^{1}$ and \\ Jérôme $\quad$ Casas $^{2,3}$ \\ ${ }^{1}$ Entomology Department, North Dakota State \\ University, PO Box 6050, Fargo, North Dakota \\ 58108-6050, U.S.A and \\ 2 Université de Tours, Institut de Recherche sur la \\ Biologie de l'Insecte, IRBI-UMR CNRS 7261, 37200 \\ Tours, FRANCE \\ ${ }^{3}$ Institut Universitaire de France, IUF, Paris, \\ FRANCE
}




\begin{abstract}
Moths are exemplars of chemical communication, especially with regard to specificity and minute amounts used. Yet, little is known about how females manage synthesis and storage of pheromone to maintain release rates attractive to conspecific males and why such small amounts are used. We developed, for the first time, a quantitative model, based on an extensive empirical data set, describing the dynamical relationship among synthesis, storage (titer) and release of pheromone over time in a moth (Heliothis virescens). The model is compartmental, with one major state variable (titer), one timevarying (synthesis), and two constant (catabolism and release) rates. The model was a good fit, suggesting it accounted for the major processes. Overall, we found the relatively small amounts of pheromone stored and released were largely a function of high catabolism rather than a low rate of synthesis. A paradigm shift may be necessary to understand the low amounts released by female moths, away from the small quantities synthesized to the (relatively) large amounts catabolized. Future research on pheromone quantity should focus on structural and physicochemical processes that limit storage and release rate quantities. To our knowledge, this is the first time that pheromone gland function has been modeled for any animal.
\end{abstract}

Key words: chemical communication; tracer-tracee analysis; compartmental model; Lepidoptera; Noctuidae

\title{
Introduction
}

Pheromones mediate a wide range of intraspecific behaviors
in animals, including mating, aggregation, sociality, alarm,
trail following and resource partitioning. These pheromones are
made and/or stored in an exocrine gland before secretion
to the body surface, or directly to the environment,
where they are detected by conspecifics (Johansson and Jones
2007; Wyatt 2014). While much work has focused on characterizing


the specificity of pheromones, the proximate mechanisms that modulate the quantities of pheromone synthesized, stored and secreted over time and space have largelybeen ignored (Foster 2016; Johansson and Jones 2007;

Umbers et al. 2015).

Probably the most widelystudied example of pheromones in animals is that of the sex pheromones of moths (Allison and Cardé 2016a; Johansson and Jones 2007; Wyatt 2014), which are exemplars of highly specific communication involving minute chemical quantities (Cardéand Baker 1984; Foster 2016; Greenfield 1981; Umbers et al. 2015). Typically, it is femalemoths that release the sex pheromone that may elicit flight responses from conspecific males over distances of tens to hundreds of meters (Cardé2016).Synthesis and release occur in an exocrine gland, usually located between the $8^{\text {th }}$ and $9^{\text {th }}$ abdominal segments (Foster 2016; Jurenka 2017; Ma and Ramaswamy 2003), over a specific period of the day (Groot 2014). Although the two physiologies temporally overlap (Groot 2014; Jurenka 2017), they are controlled by distinct mechanisms. In many species, synthesis is controlled by release of the pheromone biosynthesis activating neuropeptide(PBAN) (Jurenka 2017), 
whereas release, usually associated with an overt

behavior termed “calling" (Allison and Cardé 2016b),

appears to be controlled either directly from terminal

nerve input (Christensen et al. 1994) or, indirectly through

rhythmic muscular squeezing of the gland forcing

pheromone to the cuticular surface (Rainaet al. 2000;

Solari et al. 2007). The small quantities of pheromone

released by femalemoths must relate to the quantities of

pheromone synthesized and available for release; yet little

is knownabout the quantitative relationship between these

physiologies for any species of moth (Cardéand Baker

1984; Foster 2016; Umbers et al. 2015). In part, this

is due to the widespread use of gland titer, the amount

stored in or on the gland, as an interchangeable proxy

(e.g., Symonds et al. 2012) for both pheromone synthesis

and release (Foster 2016). While titer must relate to

both, as it resultsfrom the differential between cumulative

synthesis and usage over time, any relationship is likely to

be both time-dependent and non-linear.

Recently (Foster and Anderson 2011), we determined

pheromone synthesis rate in a moth for the first time,

by feeding femaleHeliothis virescens (Fabricius) (family: 


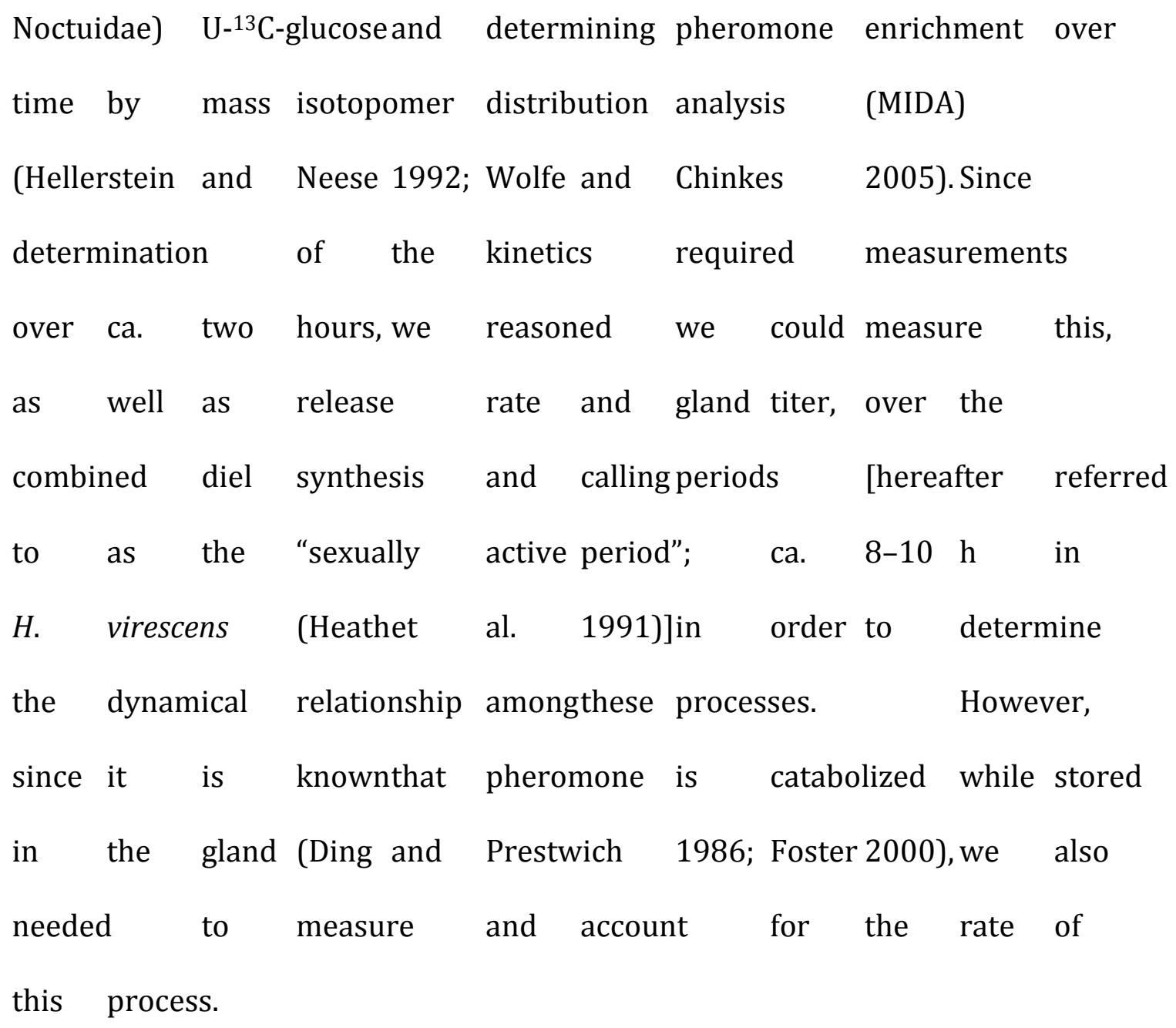

In this paper, we develop a compartmental model,based on an extensive empirical data set, describing the dynamical relationship amongsynthesis, titer, catabolism and release of sex pheromone in the moth $H$. virescens. In contrast to the assumption that costs of pheromone synthesis may limit the quantity of pheromone released (Harari et al. 2011; Johansson and Jones 2007; Umbers et al. 2015), we show that most pheromone synthesized is actually 
catabolized in the gland. Consequently, it is the amount catabolized, not synthesized that principally shapesgland titer, and limits release rate, across the sexually active period.

\section{Methods and Materials}

Conceptual Model and Biological System. We incorporated all major known processes and state variables of import to moth sex synthesis and release into our conceptual model, namely rate of synthesis (Foster and Anderson 2011; Foster et al. 2017), titer (Foster 2016), catabolism (Ding and Prestwich 1986), and release rate (Heath et al. 1991). Thus, we developed a compartmental model with one major state variable, gland titer, one input, synthesis rate, and two outputs, catabolic and release rates (Fig. 1a). We chose $H$. virescens (Fabricius) as our subject because it has a typical moth sex pheromone system, utilizing the fatty acid-derived "Type 1" moth sex pheromone components, $(Z)$ 11hexadecenal (Z11-16:Ald) and (Z)-9-tetradecenal (Z9-14:Ald) (Heath et al. 1991; Roelofs et al. 1974), and it is the only moth for which rates of pheromone synthesis have been quantified (Foster and Anderson 2011; Foster et al. 2017).

Insects. Our $H$. virescens colony was established from one previously at USDA-ARS, Fargo, and later supplemented with insects supplied by Dr. F. Gould, North Carolina State University. Larvae were maintained at $25^{\circ} \mathrm{C}$ under a 16:8 L:D photoperiod (i.e., a 480 min scotophase) and fed on a wheatgerm/casein diet. Pupae were sexed and female 
pupae placed in separate containers under the same environmental conditions as larvae. Each day, newly emerged adult females were collected and held without access to food until used in experiments. For model development, we used 2-d-old females (i.e., $2 \mathrm{~d}$ after eclosion), while for model testing we used 1- and 3-d-old females.

Titer. The gland of an individual female was dissected, and extracted in $n$-heptane, along with $25 \mathrm{ng}$ of (Z)-11-tetradecenal as internal standard, for at least $1 \mathrm{~h}$ at ambient temperature. We quantified only Z11-16:Ald, since this is $>90 \%$ of the total pheromone (Heathet al. 1991), and we wished to model quantity, not blend ratio. For titer determination, we used gas chromatography/mass spectrometry (GC/MS; see supplementary) and monitored $\mathrm{m} / \mathrm{z}, 192$ and 220 for Z11-14:Ald (internal standard), and Z11-16:Ald, respectively.

We analyzed 2-d-old females every $120 \mathrm{~min}(\mathrm{~N}=10$ for each time point), starting 120 min before, through to the end of, the scotophase. Females were also analyzed $360 \mathrm{~min}$ into the following photophase, when they are not producing significant amounts of pheromone (Foster and Anderson 2011). We also analyzed 1- and 3-d-old females $(\mathrm{N}$

$=10$ for each time point) at the beginning and middle of the scotophase.

Synthesis rate. Females were fed $\mathrm{U}_{-}{ }^{13} \mathrm{C}$-glucose (99\% enriched, Cambridge Isotope Laboratories, Cambridge, MA), and synthesis rate of Z11-16:Ald determined using MIDA (Hellerstein and Neese 1992; Wolfe and Chinkes 2005), as previously described 
(Foster and Anderson 2011); see supplementary material for a more detailed description. Briefly, females that ingested $25 \mu \mathrm{l}$ of a $10 \%(\mathrm{w} / \mathrm{v}) \mathrm{U}-{ }^{13} \mathrm{C}$-glucose solution were analyzed (for titer) at various times over the next 120 min (including before feeding; i.e., at $\mathrm{t}=0$ ). U- ${ }^{13} \mathrm{C}$-glucose is rapidly absorbed, glycolyzed and oxidized to acetyl CoA, which is used for de novo pheromone biosynthesis (Foster and Anderson 2011). For MIDA, we monitored $\mathrm{m} / \mathrm{z} 220,222$, and 224, representing unlabeled (M+0), singly labeled $(\mathrm{M}+1)\left(\right.$ one ${ }^{13} \mathrm{C}_{2}$ unit) and doubly labeled $(\mathrm{M}+2)$ isotopomers of Z11-16:Ald. Enrichment of pheromone reached equilibrium 90 min after feeding for all time periods; therefore, we used precursor enrichment of individual females 90 min after feeding to calculate individual fractional synthetic rates (FSR; see supplementary). Synthesis rate for each time period was calculated by multiplying FSR by pool size (titer at $t=0$ for each time period).

For 2-d-old females, synthesis rate was determined every $120 \mathrm{~min}$ of the scotophase (i.e., starting at $\min 0,120,240$ and 360) and also starting at $\min 360$ of the photophase. For 1and 3-d-old females, synthesis rate was determined at min 0 and 240 of the scotophase only. For each time point, 5-10 individual females were analyzed. Errors were calculated as standard errors of the product of two random variables, titer and FSR, following Lynch and Walsh (1998).

Release rate. Just prior to the scotophase start, two females of the same age (1, 2 or $3 \mathrm{~d})$ were placed inside a glass chamber $(400 \mathrm{ml})$ through which charcoal-filtered air flowed at $300 \mathrm{ml} . \mathrm{min}^{-1}$. Pheromone released was collected on Tenax TA (400 mg; 60-80 mesh, 
Supelco, Bellefonte, PA), inside a Pasteur pipet with glass wool at the ends. Pheromone was collected for 120 min periods throughout the scotophase, commencing at the beginning (with all four time periods collected for the same pair of females). In addition, for 2-d-old females, we collected for $120 \mathrm{~min}$, starting at min 60 and 180 of the scotophase (again both times from the same pair). Pheromone was desorbed with nhexane, and analyzed by GC/MS (see titer method). Nine pairs of females were sampled for each $120 \mathrm{~min}$ period.

Glandular catabolism. Females ( 2 d only; 6-15 per time point) were decapitated midway through the scotophase (when titer was high) and titer determined by GC/MS at various times over the next $240 \mathrm{~min}$. To confirm that decapitation also stops pheromone release (the other possible fate of pheromone), we collected pheromone released from pairs of females $(\mathrm{N}=4)$ immediately after decapitation (120 min into the scotophase) over the next $240 \mathrm{~min}$.

Calling periodicity. Individual females ( $\mathrm{N}=20$ for each of 1,2 and $3 \mathrm{~d}$-old) were placed in clear glass vials $(25 \times 75 \mathrm{~mm})$ just prior to the start of the scotophase, and observed every 30 min over the entire scotophase for whether or not they exhibited calling behavior. A red-filtered light (10 lux) was used to aid observations. Data were summarized as proportions of females exhibiting calling during each 60 min period.

Model. In addition to our major state variable (titer), input (pheromone synthetic rate), and outputs (catabolic and release rates) (Fig. 1a), we defined further state variables, the integrals of synthesis, release and catabolic rates. According to the usual nomenclature of 
compartmental models (DiStefano III 2013), we called a and d the release and catabolic rate constants, with time ${ }^{-1}$ units. Thus, a pheromone release or catabolic rate (mass per unit time) refers to the appropriate rate constant multiplied by titer, T. By contrast, the time-dependent pheromone synthetic rate is given directly in mass per unit time, as we have no estimate for the upstream compartment amounts. All rates are expressed as ng. $\min ^{-1}$ Z11-16:Ald.

The equations in the model were:

(1) Titer: $\quad d T / d t=b(t)-(d * T)-(a * T)$

(2) Synthesis: $d S / d t=b(t)$

(3) Catabolism: $\mathrm{dD} / \mathrm{dt}=\mathrm{d} * \mathrm{~T}$

(4) Release: $\mathrm{dR} / \mathrm{dt}=\mathrm{a} * \mathrm{~T}$

In the implementation of the model using R (R Core Team 2013), a linear interpolation method was used (function approxfunction in Soetaert, Cash \& Mazzia 2012, page 57), due to the different time bases (spans) in the experiments. For example, titer was an instantaneous measure (e.g., at $\mathrm{t}=0 \mathrm{~min}$ ), whereas synthesis and release rate measurements were made over $2 \mathrm{~h}$ periods (e.g., from $\mathrm{t}=0-120 \mathrm{~min}$ ). Since we had to choose the same instant in time for the multiplication of titer and FSR to estimate synthetic rate, we chose the midpoint of these determinations (in our example, $t=60 \mathrm{~min}$ ) as the nominal value and used titer at the start of that period (i.e., before label had been ingested). We also used a mid-time point for pheromone release. In practice, this is reasonable for FSR, since we used precursor enrichment at $\mathrm{t}=90 \mathrm{~min}$ and the rate of change of enrichment over the period of 0-60 min for the calculation (see supplementary); however, it did introduce some inaccuracy in titer (real titer at $\mathrm{t}=0$ is 
different from real titer at $60 \mathrm{~min}$ ). Since titer data were more variable than other data sets, we screened for outliers using the Shapiro test at 0.05 level and quantile plots for normal distributed values; two outliers were excluded from the analysis.

Our model is composite and dynamical, so the usual measures of overall goodness of fit do not operate; therefore, one has to use more qualitative assessments, such as the presence of biases, qualitative trends, behavior of the variances, consistency of assumptions, structural robustness or independent data sets (Goriely 2018, Haefner 2005, Weisberg 2013). In this respect, the pheromone release rates of days 1 and 3 are true independent data sets for testing the model. The cumulative released amounts were well predicted for these days, a strong test of the model.

We did not conduct a systematic sensitivity analysis of the model. Rather, we explored the consequences of changing parameter values to represent some biologically interesting scenarios.

The outputs of these simulations are reported in appropriate places in the Discussion.

\section{Results}

\begin{tabular}{|c|c|c|c|c|c|c|c|c|c|}
\hline \multicolumn{2}{|c|}{ Synthesis } & rate. & \multicolumn{2}{|c|}{ Synthesis } & rate & in & 2-d-old & females & \multirow[t]{2}{*}{ showed } \\
\hline $\mathrm{a}$ & rapid & rise & aroun & & the & sta & the & scotophase, & \\
\hline \multicolumn{2}{|c|}{ maintained } & its & level, & \multicolumn{3}{|c|}{ before declining } & rapidly & the & end \\
\hline of & the & scotop & hase & (Fig. & 1b). & It & likely maint & ained & low \\
\hline \multicolumn{2}{|c|}{ (basal) } & rate & for & most & of & the & subsequent & photophase, & as \\
\hline \multicolumn{2}{|c|}{ nominally } & 420 & $\min$ & into & the & \multicolumn{2}{|c|}{ photophase, the } & synthesis & rate \\
\hline was & 12.6 & ng.h-1 & (data & not & used & in & model). & While our & data \\
\hline
\end{tabular}


for determination, the pattern suggests a mostly stepwise on/off (or very low) synthesis, consistent with diel release of PBAN controlling the activity of an enzyme(s) in the pathway (Jurenka 2017).

For the two periods measured (nominally 60 and 300 min into the scotophase) for each of 1- and 3-d-old females, there was a similar increase in synthesis rate as observed for $2 \mathrm{~d}$ females over the same times, although synthesis rate declined with increasing age. The data used to calculate synthetic rate are given in supplementary

Table 1.

For the model, we fitted three different hyperbolic Michaelis-Menten equations to the observed rates for each of the three days (Fig. 1b). The number of sampling times is too low to apply non-linear fits (four sampling points for day 2, and only two for days 1 and day 3), so we used a linear fit on linearized data, as done in Crawley (2007, page 203). Note that this technique required us to assume that the plateau was reached after $5 \mathrm{~h}$ in the scotophase and forces the fit through this point. Hence, no goodness of fit can be used.

Release rate. Release rate by 2-d-old females increased through the first hours of the scotophase. 


\begin{tabular}{|c|c|c|c|c|c|c|c|c|}
\hline However, & after reach & & $\mathrm{a}$ & peak & nomi & ially & $180 \min$ & into \\
\hline sco & hase, relea & & rate & declin & & slightly & and & held \\
\hline steady for & rema & nder & of & the & scoto & hase & (when meas & Irements \\
\hline ceased) & (Fig. & & Thre & -d-old & femal & & showed & $\mathrm{a}$ \\
\hline similar & release & rate & patte & & over & time & that & of \\
\hline 2-d-old & females. & By & contr & ast, & 1 & d-old & females & had \\
\hline peak rele & rate & som & what & later & (ca. & 360 & $\min$ ) in & the \\
\hline scotophase & gene & & fems & es relea & ed ph & comone a & at a lower $r$ & with \\
\hline
\end{tabular}

The plotted estimate of the release rate constant of $2 \mathrm{~d}$ females showed high variability and no clear trend, so we estimated it at $0.0028 \mathrm{~min}^{-1}$ (S.E. $0.0005, \mathrm{n}=4$ ), and assumed it constant over the three days.

Glandular catabolism. Following decapitation, titer stayed briefly at the
same level before declining very rapidly, such that 240 min
after decapitation, it was roughly $10 \%$ that prior to
decapitation (Fig. 1d). Decapitated females did not release
detectable levels of pheromone over the 4 h following
decapitation.

An exponential decline $[\mathrm{y}=\log (-0.012 \mathrm{x}+4.24), 92$ d.f., $\mathrm{P}<0.0001$, residual standard error: 0.8095] for the data of decapitated females (Fig. 1d) gave a good fit. The residual 
analysis shows, however, a sharper decline very early on, not captured by the exponential decrease. The degradation rate constant $\quad=0.012 \mathrm{~min}^{-1}$ (S.E. 0.0013), and was assumed to be constant over the three days.

Titer. Two d-old females showed a typicaltiter pattern, observed in this (Foster

2005; Raina et al. 1986) and other species (Groot2014) of moths,consisting of a low (basal) titer prior to the start of the scotophase, rising to a peak around midscotophase and then dropping rapidly back to the basal level by the end of the scotophase and through the following photophase (Fig. 2b; supplementary Table 1). The dynamics of titer in the model showed a convex curve when titer was increasing and a concave one when decreasing. Only two time points $(0$ and 240 min in the scotophase) were measured for both 1 and 3 d-old females, and these showed a similar pattern to 2 d-old females (Fig. 2a,c), Titer generally decreased with increasing age (Fig. 2a,b,c). Titer showed greater change throughout the scotophase than did synthesis or release rates.

Model testingand exploration. The predicted titers (Fig. 2a-c), as well as cumulative pheromone released over each of the three days, closely followed the 
observed values, such that we are confident that the model contains the major processes contributing pheromone quantity management in the gland. A comparison of the rate of synthesis with the rate of disappearance (usage), defined as the sum of the rates of release and catabolism, shows that synthesis is greater than usage, particularly through the first half of the scotophase (supplementary Fig. 1), allowing the titer increase observed during this period (Fig. 2). Toward the end of the scotophase, when synthesis had stopped, the large negative differential resulted in the sharp decrease in titer observed (Fig. 2). Figure 3

(along with Fig. 2d), summarizes all the predicted processes, enabling a clear comparison of fluxes and age-related processes; note the large proportion of pheromone degraded compared to that released.

Calling periodicity. Females of all three ages showed similar patterns of calling through the first 300 min of the scotophase. Basically, a small proportion of females called early, with most calling between 120-300 min (Fig. 4). Peak calling occurred earlier in the sexually active period with increasing age, a phenomenon noted in other species (e.g., Webster and Cardé 1982). A high proportion of 1-d-old females called past hour 5 of the scotophase, in contrast to the other two ages.

\section{Discussion}

To maximize fecundity, a female moth must mate soon after becoming sexually mature, as delays can be deleterious (Umbers et al. 2015). The synthesis and release of sex pheromone are crucial for ensuring rapid mating of sexually mature females (Allison and 
Cardé 2016a). For the first time, we have collected and modeled dynamic data on pheromone synthesis, storage, catabolism and release in a moth, to understand how the system manages attractive pheromone release rates across a daily calling period. The data fit was good, suggesting that we accounted for the major processes influencing gland quantity management.

Pheromone catabolism modulates gland titer. Catabolism is the primary use of pheromone, accounting for roughly $80 \%$ of pheromone produced over the course of a sexually active period. Consequently, it has a much greater affect on shaping pheromone titer over time than does release, and effectively limits peak titer. Without catabolism, model simulations predict $1 \mathrm{~d}$ females attaining a peak titer of $410 \mathrm{ng}$, a value well in excess of any we have observed. However, we observed two $1 \mathrm{~d}$ females with titers of ca. $200 \mathrm{ng}$ (these appeared to fit a different distribution and were excluded as outliers from our analyses). Interestingly, the model predicts a similar value (170 $\mathrm{ng})$ for $1 \mathrm{~d}$ females if they catabolize, but do not release, pheromone. While, these unusual titers could also result from reduced catabolism or increased synthesis, this prediction suggests that some females, which on the basis of titer might be considered "high releasers", may not in fact actually release (much) pheromone.

In endocrine systems, hormone titers are controlled by a combination of mechanisms (Molina 2013). For example, in insects, juvenile hormone titers are controlled by feedback mechanisms controlling synthesis, catabolism, sequestration, and protection from catabolism (Goodman and Cusson 2012; Nijhout and Reed 2008). In comparison, titer in the exocrine pheromone system of $H$. virescens is controlled predominantly by 
usage (release and catabolism), with the system appearing to lack feedback control over synthesis, as evidenced by a fairly constant synthesis rate regardless of titer profile. Indeed, the only control of synthesis appears to be the circadian release/non-release of PBAN, which probably defines the period of synthesis but not the degree (Groot 2014; Jurenka 2017). The lack of fine control of synthesis throughout this period may follow from the pheromone's target being exogenous (i.e., to males), rather than endogenous, to the producer (female).

Why modulate titer? It is generally accepted that the release of greater quantities of pheromone should result in females attracting more males over greater distances, and hence is likely to enhance fitness (Baker and Roelofs 1981; Cardé 2016; Foster and Johnson 2011; Greenfield 1981; Symonds et al. 2012; Umbers et al. 2015). Why, then, do females need a titer control that lowers amounts of pheromone available for release? One possibility is that high titers of pheromone might be deleterious to gland cell function. This was suggested for species that use aldehyde components (like H. virescens) following the identification of non-specific oxidases that converts alcohols to aldehydes in the cuticular layer of the gland (Fang et al. 1995; Teal and Tumlinson 1988). However, it fails to explain why species that do not use aldehyde components also catabolize pheromone [e.g., (Foster 2000)]. We think symmorphosis a more likely explanation for titer modulation; i.e., that the gland is structurally incapable of release rates substantially greater than the maximal ones observed (in $1 \mathrm{~d}$ females). Catabolism thus prevents the gland from accumulating excess pheromone, which it cannot release during the calling period. For example, without catabolism, our model predicts that $1 \mathrm{~d}$ females would have a titer of $315 \mathrm{ng}$ at the end of the calling period. Such large amounts of pheromone 
remaining after calling had ceased might result in (accidental) further release, subjecting refractory and reproducing females to continued harassment by males.

Assuming a passive release of pheromone (e.g., Solari et al. 2007), the evaporation rate of a given component (of specific vapor pressure) will depend upon flux to the cuticular surface and the area of the gland over which pheromone is distributed, as well as environmental factors such as temperature and windspeed (Nielsen et al. 1995). Little is known about pheromone movement through the gland or its distribution on the surface, precluding calculations of theoretical release rates based on vapor pressures. However, one or both could be limiting, meaning that increased synthesis or indeed reduced catabolism might result in increased titer but not in increased release rate. Studies quantifying pheromone glandular fluxes and surface distribution, in combination with both theoretical and empirical determinations of release, are needed to understand how gland structure may determine pheromone release rate.

A relatively high rate of synthesis, combined with catabolism, allows titer to build up rapidly when synthesis starts and decline rapidly once synthesis stops, helping synchronize pheromone availability/non-availability with calling periodicity. A rough synchronization of pheromone availability with calling periodicity is suggested by our data. At the start of the scotophase, when pheromone is being synthesized and titer increasing, high proportions of females of all three ages call. However, near the end of the scotophase, when synthesis has stopped, only $1 \mathrm{~d}$ females have high titers, and only they are still calling in high proportions. The slightly earlier peak in calling with increasing age is consistent with other studies (Umbers et al. 2015). We note, however, that females were not fed in this experiment. Just as starvation can influence pheromone 
titer (Foster 2009; Foster and Johnson 2010), it might also influence calling behavior and release rate.

Conclusion. A reason often cited for why moths release small amounts of chemicals is that excess synthesis represents some 'cost' to a female (Cardé and Baker 1984; Harari and Steinitz 2013; Johansson and Jones 2007; Symonds et al. 2012). Our work demonstrates that catabolism, not release, is the primary fate of most pheromone synthesized by $H$. virescens females and probably for many other moth species. This suggests a paradigm shift may be necessary to understand the low amounts released, away from focusing on the small quantities synthesized to focus on the (relatively) large amounts catabolized. Our model helps explain how producing a large excess of pheromone over that released is not the outcome of a maladaptive process, but is constitutive to the design of a control mechanism that both limits storage and synchronizes availability/non-availability of pheromone with need (release through calling). Our model forms the basis of future work looking at neglected gland processes that determine release rate, including catabolism and cell to surface pheromone flux, as well as structural limitations including pore diameter and density and cuticular pheromone distribution. To our knowledge, this is the first model containing the major known physiological processes of a pheromone gland in any animal, making it a framework for the quantitative functioning of exocrine glands in species from moths to mammals. 


\section{Acknowledgments}

This work was funded in part by a United States Department of Agriculture Hatch Project ND02388 (to SPF). The purchase of the GC/MS system was funded in part by a United States Department of Agriculture-National Institute of Food and Agriculture Instrument Grant, 2015-07238 (to SPF). We thank Le Studium Loire Valley Institute for Advanced Studies and Dr. D. Giron for supporting the visit of SPF to Tours, where this collaboration was initiated. We also thank Dr. T. Zemb (ICSM, Site de Marcoule) and the INOV group at IRBI for discussions and comments on the work. 


\section{References Cited}

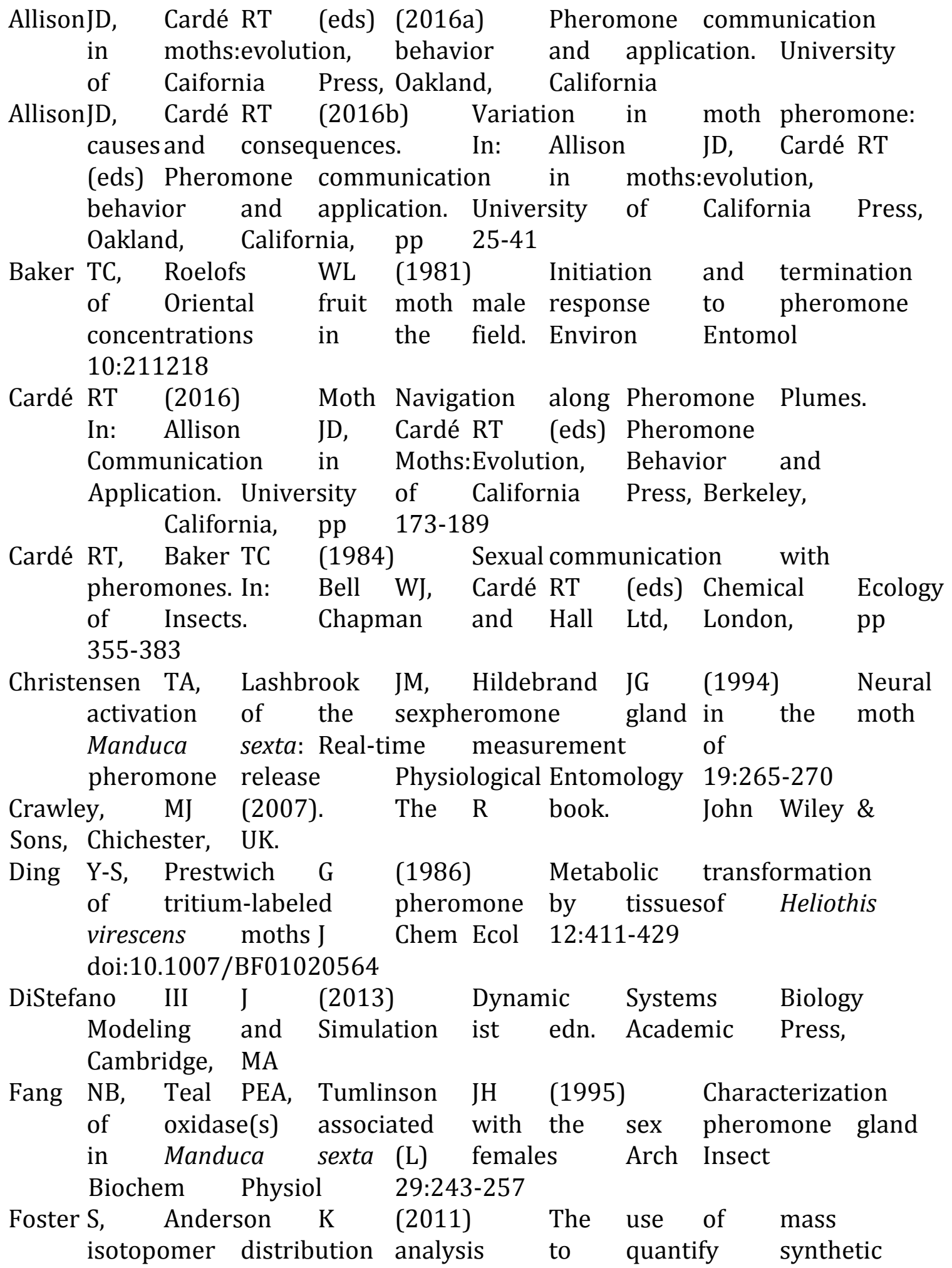


rates of sex pheromone in the moth Heliothis virescens. J Chem Ecol 37:1208-1210

Foster S, Johnson C (2011) Signal honesty through differential quantity in the female-produced sex pheromone of the moth Heliothis virescens. J Chem Ecol 37:717-723

Foster SP (2000) The periodicity of sex pheromone biosynthesis, release and degradation in the lightbrown apple moth, Epiphyas postvittana (Walker). Arch Insect Biochem Physiol 43:125-136

Foster SP (2005) Lipid analysis of the sex pheromone gland of the moth Heliothis virescens. Arch Insect Biochem Physiol 59:80-90 doi:DOI: 10.1002/arch.20058

Foster SP (2009) Sugar feeding via trehalose haemolymph concentration affects sex pheromone production in mated Heliothis virescens moths J Exp Biol

212:2789-2794 doi:doi: 10.1242/jeb.030676

Foster SP (2016) Toward a quantitative paradigm for sex pheromone production in moths.In: Allison JD, Cardé RT (eds) Pheromone communication in moths: evolution, behavior and application. University of California Press, Oakland, CA, pp 113-126

Foster SP, Anderson KG, Casas J (2017) Sex pheromone in the moth Heliothis virescens is produced as a mixture of two pools: de novo and via precursor storage in glycerolipids Insect Biochemistry and Molecular Biology 87:26-34 doi:http://dx.doi.org/10.1016/j.ibmb.2017.06.004

Foster SP, Johnson CP (2010) Feeding and hemolymph trehalose concentration influence sex pheromone production in virgin Heliothis virescens moths J Insect Physiol 56:1617-1623 doi:doi:10.1016/j.jinsphys.2010.06.002

Goodman WG, Cusson M (2012) The Juvenile Hormones In: GilbertLI (ed) Insect Endocrinology. Academic Press, San Diego, pp 305-394. doi:https://doi.org/10.1016/B978-0-12-384749-2.10008-1

Goriely, A. (2018). Applied Mathematics: A Very Short Introduction. Oxford University Press. Oxford, U.K.

Greenfield MD (1981) Moth sex pheromones: an evolutionary perspective Florida Entomol 64:4-17

Groot AT (2014) Circadian rhythms of sexual activities in moths: a review Frontiers in Ecology and Evolution 2 doi:10.3389/fevo.2014.00043

Haefner, J. W. (2005). Modeling Biological Systems: Principles and Applications. 
Springer Science \& Business Media, Dordrecht, The Netherlands.

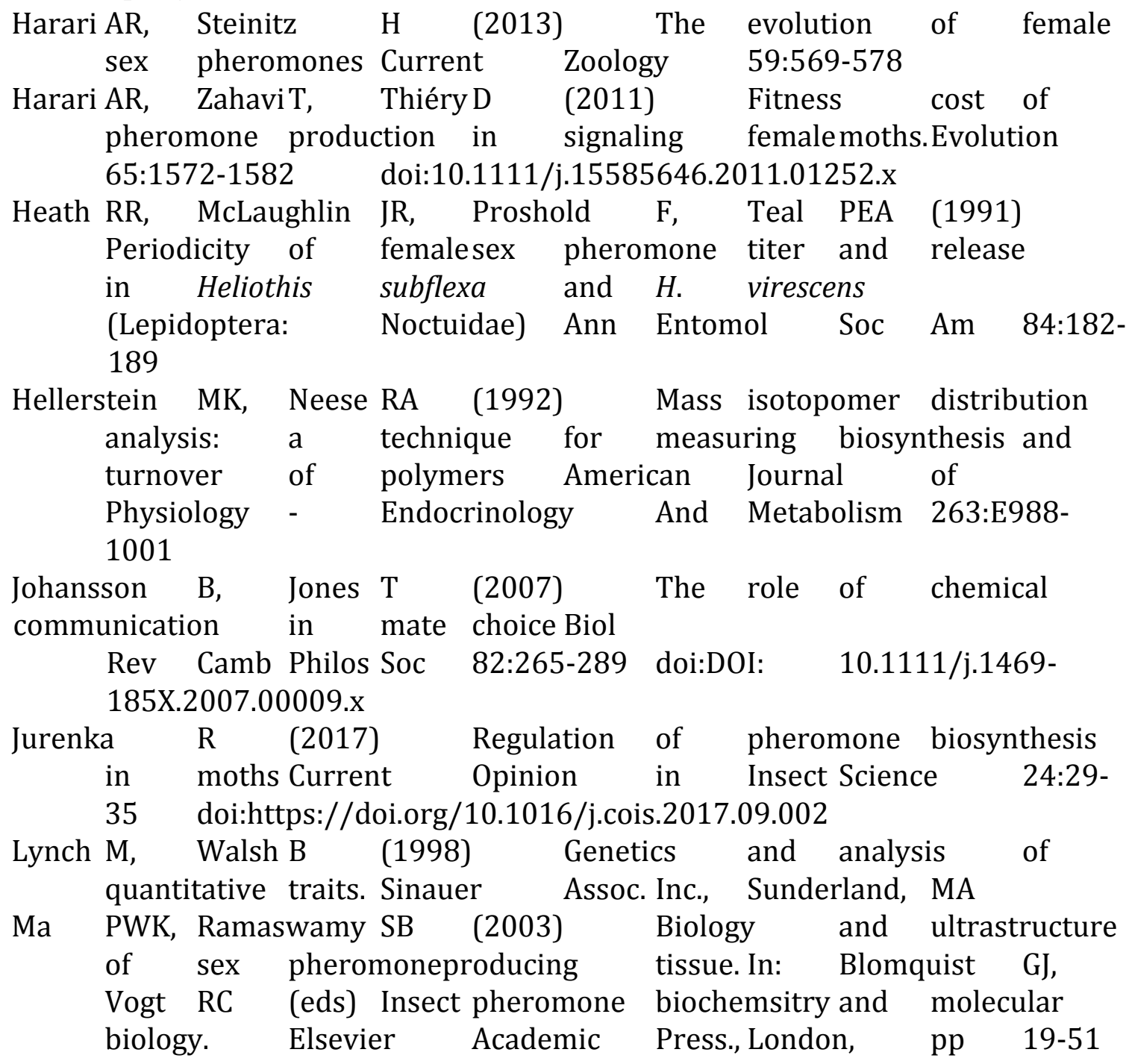
MolinaPE (2013) Endocrine physiology. 4th edn. McGraw-Hill, New York, NY

Nielsen F, Olsen E, Fredenslund A (1995) Prediction of isothermal evaporation rates of pure volatile organic compounds in occupational environments-A theoretical approach based on laminar boundary layer theory The Annalsof Occupational Hygiene 39:497-511 doi:https://doi.org/10.1016/00034878(95)00032-A

Nijhout HF, Reed MC (2008) A mathematicalmodel for the regulation of juvenile hormone titers $\mathrm{J}$ Insect Physiol 54:255-264 doi:https://doi.org/10.1016/j.jinsphys.2007.09.008 
R Core Team (2013) R: A language and environment for statistical computing. $\mathrm{R}$ Foundation for Statistical Computing, Vienna, Austria

Raina AK, Klun JA, Stadelbacher EA (1986) Diel periodicity and effect of age and mating on femalesex pheromone titer in Heliothis zea (Lepidoptera: Noctuidae). Ann Entomol Soc Am 79:128-131

Raina AK, Wergin WP, Murphy CA, Erbe EF (2000) Structural organization of the sex pheromone gland in Helicoverpa zea in relation to pheromone production and release Arthropod Structure and Development 29:343353

Roelofs WL, Hill AS, Cardé RT, Baker TC (1974) Two sex pheromone components of the tobacco budworm moth, Heliothis virescens. Life Sci 14:1555-1562 doi:doi:10.1016/0024-3205(74)90166-0

Solari P, Crnjar R, Spiga S, Sollai G, Loy F, Masala C, Liscia A (2007) Release mechanism of sex pheromone in the femalegypsy moth Lymantria dispar:a morpho-functional approach Journal of Comparative Physiology A 193:775-

785 doi:10.1007/s00359-007-0232-z

Symonds MRE, Johnson TL, Elgar MA (2012) Pheromone production, male abundance, body size, and the evolution of elaborate antennae in moths Ecology and Evolution 2:227-246 doi:10.1002/ece3.81

Teal PEA, Tumlinson JH (1988) Properties of cuticular oxidases used for sex pheromone biosynthesis by Heliothis zea J Chem Ecol 14:2131-2145

Umbers KDL, Symonds MRE, Kokko H (2015) The Mothematics of Female Pheromone Signaling: Strategies for Aging Virgins The American Naturalist 185:417-432 doi:10.1086/679614

Webster RP, Cardé RT (1982) Relationship amongpheromone titre, calling and age in the omnivorous leafroller moth (Platynota stultana). J Insect Physiol 28:925-933

Weisberg, M. (2012). Simulation and similarity: Using models to understand the world. Oxford University Press, Oxford, U.K.

Wolfe RR, Chinkes DL (2005) Isotope Tracers in Metabolic Research, Second Edition. John Wiley \& Sons, Inc.,

Wyatt TD (2014) Pheromones and Animal Behaviour:

Chemical Signals and

Signatures. 2nd edn. Cambridge University Press, 


\section{Captions for onlineversion}

$\begin{aligned} & \text { Figure 1. } \\ & \text { virescens }\end{aligned}$ Modelfor the
and parameter $\begin{aligned} & \text { pheromone gland of Heliothis } \\ & \text { estimations for associated physiological } \\ & \text { processes } \\ & \text { through the }\end{aligned}$

occurred).

Figure2. Modeltesting. (a-c) Observed (open circles) titers of individual females and predicted titers (lines) over the scotophase (time 0-480 min), and (d) mean observed and predicted cumulative amounts of pheromone released by females for each of the three days $(1 \quad d$ black, 2 d red, $3 \mathrm{~d}$ blue). The shaded area shows when pheromone synthesis stops.

Figure3. Modelexploration. Predicted mean amounts cumulatively synthesized, (b) stored in the gland, and (c) cumulatively catabolized over the scotophase $(\min 0-480)$ for each of the three days $(1 \quad d$ black, 2 d red, $3 \mathrm{~d}$ blue). The shaded area shows when pheromone synthesis stops.

Figure4. Calling frequency of females of different age. While high proportions of females of all ages $(1 \mathrm{~d}$ black, $2 \mathrm{~d}$ red, $3 \mathrm{~d}$ blue) call in the first part of the scotophase (min 0-480), only 1 d females call in high proportions in the latter part. The shaded area shows when pheromone synthesis stopped. The colored arrows indicate approximate peaks of callingfor the three age groups.

\section{Captions for print version}


Figure1. Modelfor the pheromone gland of Heliothis virescens and parameter estimations for associated physiological processes through the scotophase $(0480 \mathrm{~min})$. The compartmental model (a) has three state variables: gland titer, release and catabolism. The pheromone synthesis rate is the only time varying parameter. The observed rates of synthesis (b) and release (c) are shownfor females age 1 (open circles), 2 (closed squares) and 3 $\begin{array}{llll}\text { (stars) days, respectively (mean } \pm & \text { S.E.M).In } & \text { (b) and (c), the }\end{array}$ shaded area shows when synthesis stops (illustrated by the low rate at $420 \mathrm{~min}$ ). (d) Time course of gland titer (plus fitted curve) after decapitation (of $2 \quad \mathrm{~d}$ females) at 240 min into the scotophase (arrow shows when decapitation occurred).

Figure2. Modeltesting. Observed (open circles) titers of individual females and predicted titers (lines) over the scotophase (time 0-480 min) for (a) 1-d-old, (b), 2d-old, and (c) 3-d-old; $\quad$ (d) mean observed and predicted cumulative amounts of pheromone released by females for each of the three days $(1 \mathrm{~d}=$ dotted line, $2 \mathrm{~d}$ $=$ solid line, $3 \mathrm{~d}=$ dashed line). The shaded area shows when pheromone synthesis stops.

Figure3. Model exploration. Predicted amounts (a) cumulatively synthesized, (b) stored in the gland, and (c) cumulatively catabolized over the scotophase (min 0-480) for each of the three days $(1 \mathrm{~d}=$ dottedline, $2 \mathrm{~d}$ $=$ solid line, $3 \mathrm{~d}=$ dashed line). The shaded area shows when pheromone synthesis stops.

Figure4. Calling frequency of females of different age. While high proportions of females of all ages $\left(\begin{array}{lll}1 & d\end{array}\right.$ $=$ dotted line, $2 \mathrm{~d}=$ solid line, $3 \mathrm{~d}=$ dashed line) call in the first part of the scotophase (min 0-480), only 1 d females call in high proportions in the latter part. $\mathrm{N}=20$ for each time point. The shaded area shows when pheromone synthesis stopped. The colored arrows indicate approximate peaks of calling for the three age groups. 


\section{Figure 1}

(a)

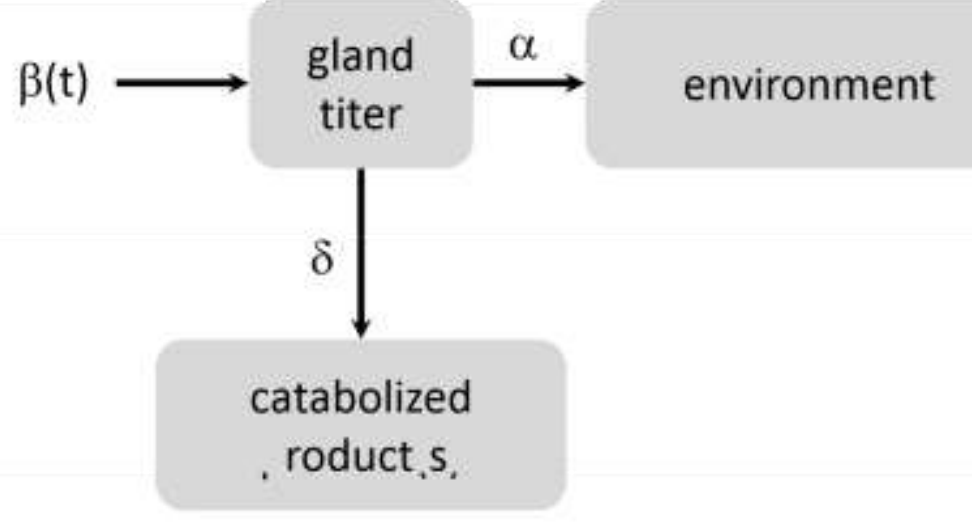

pheromone synthesis

(D)

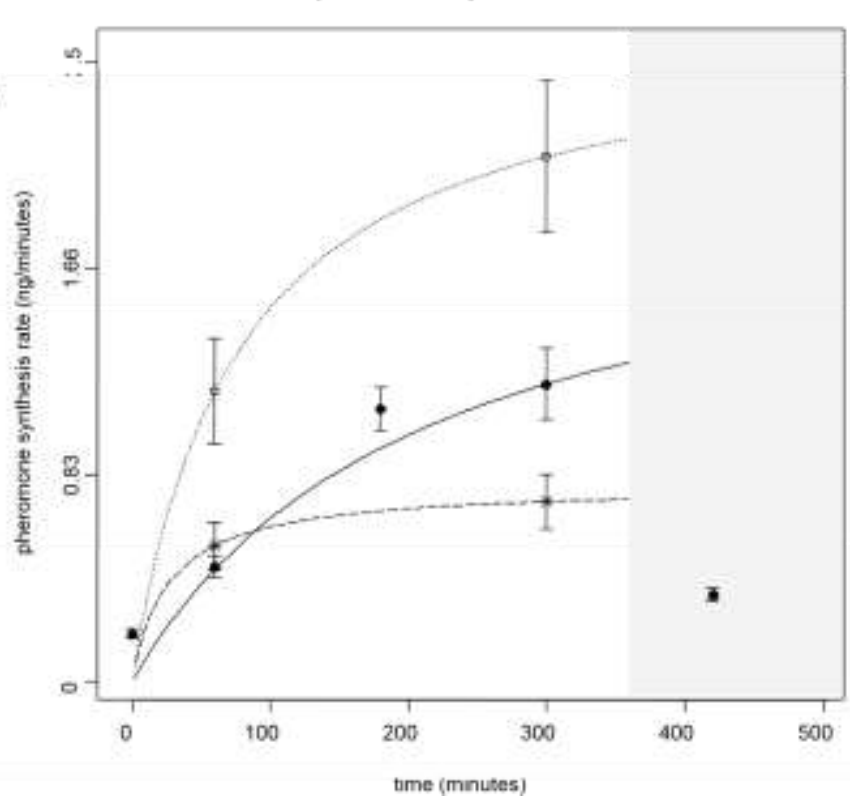

pherompheromone catabolisme degradation

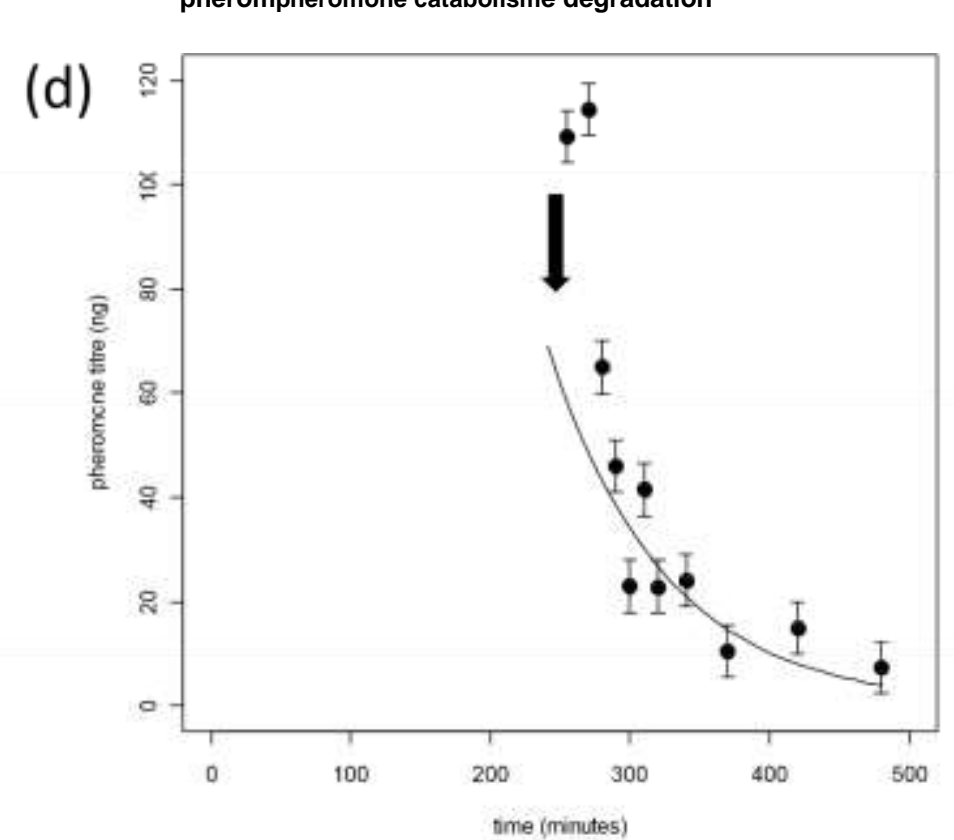

(c)

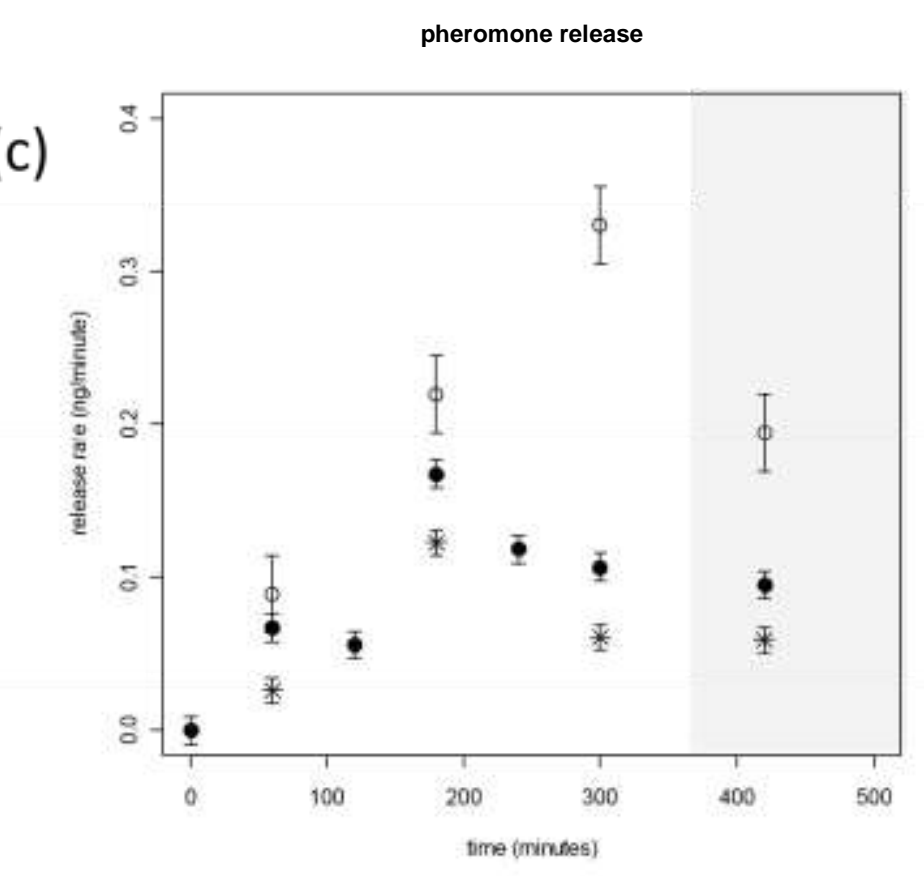

pheromone release 


\section{Figure 2}
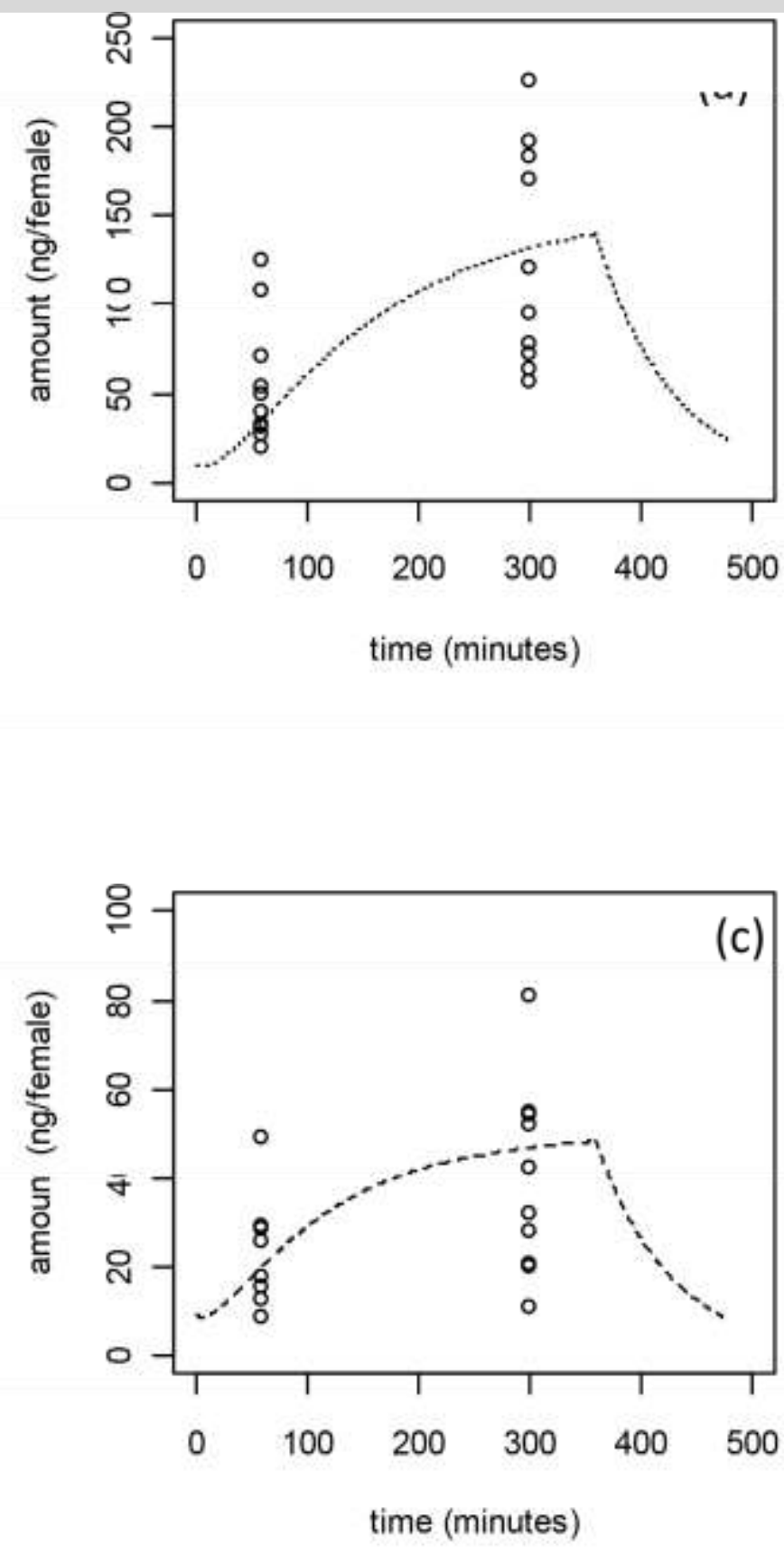

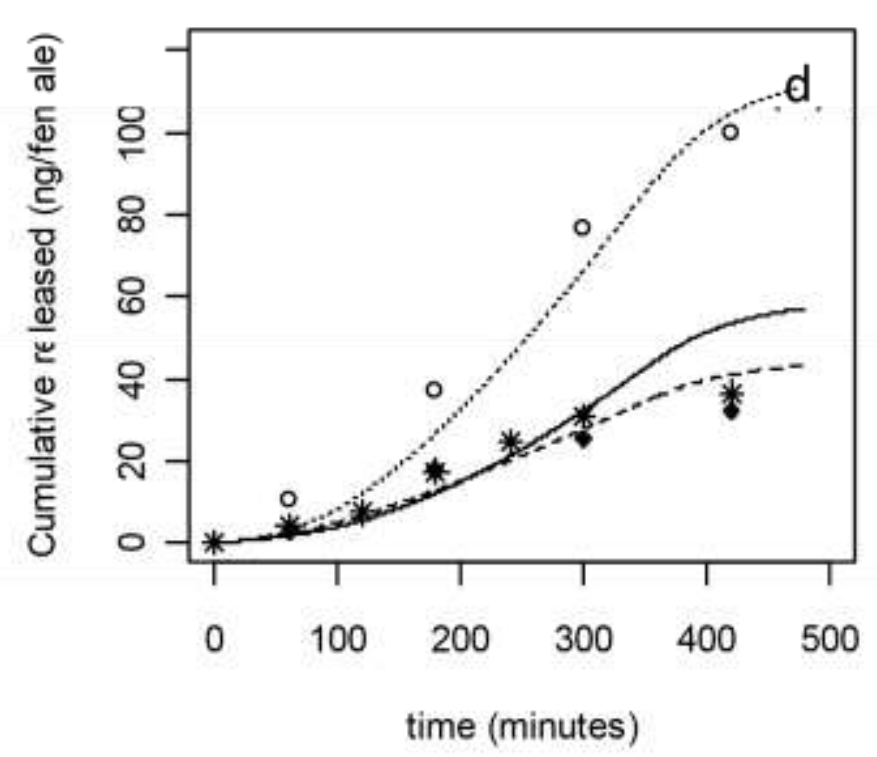




\section{Figure 3}
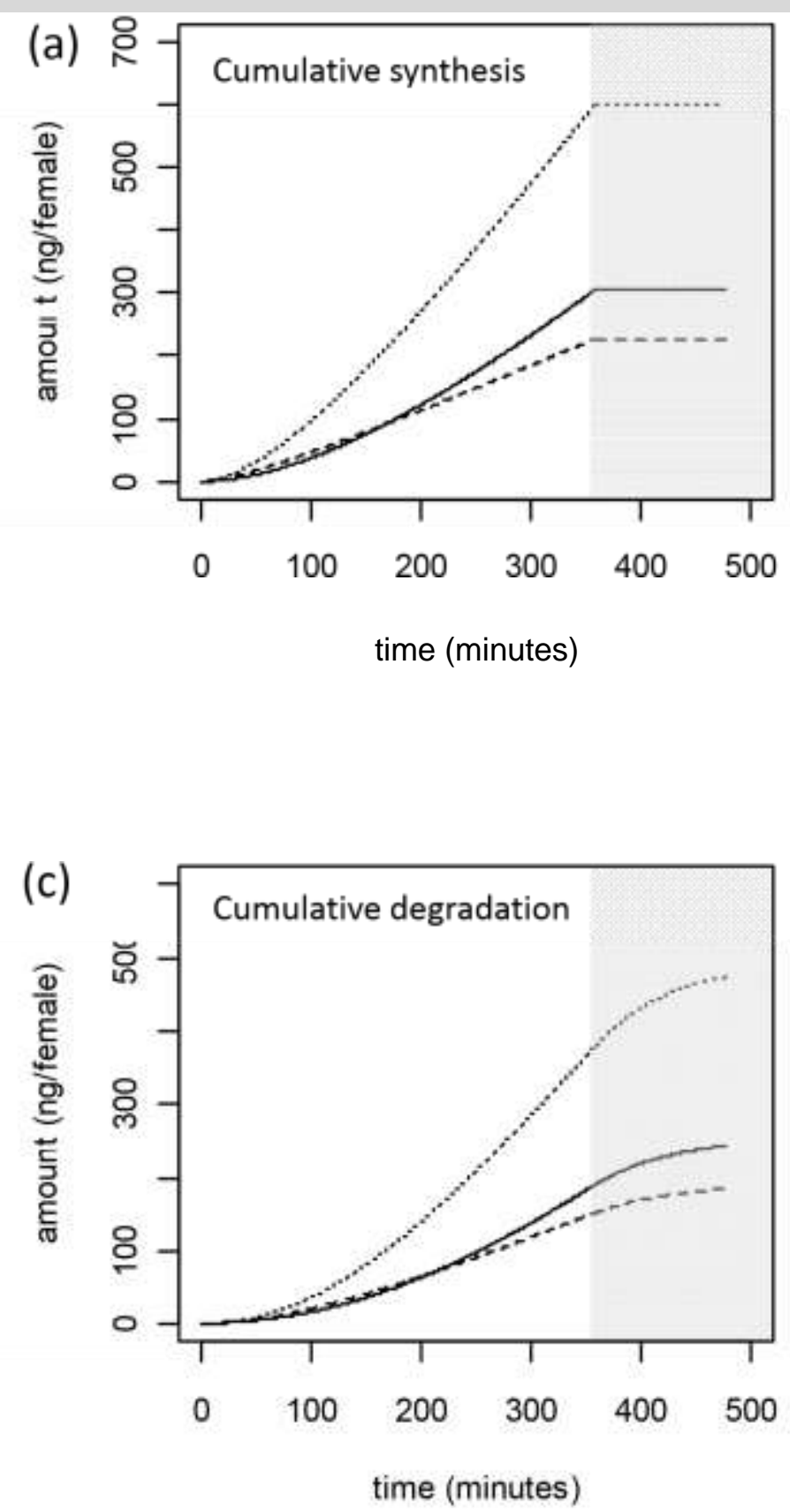

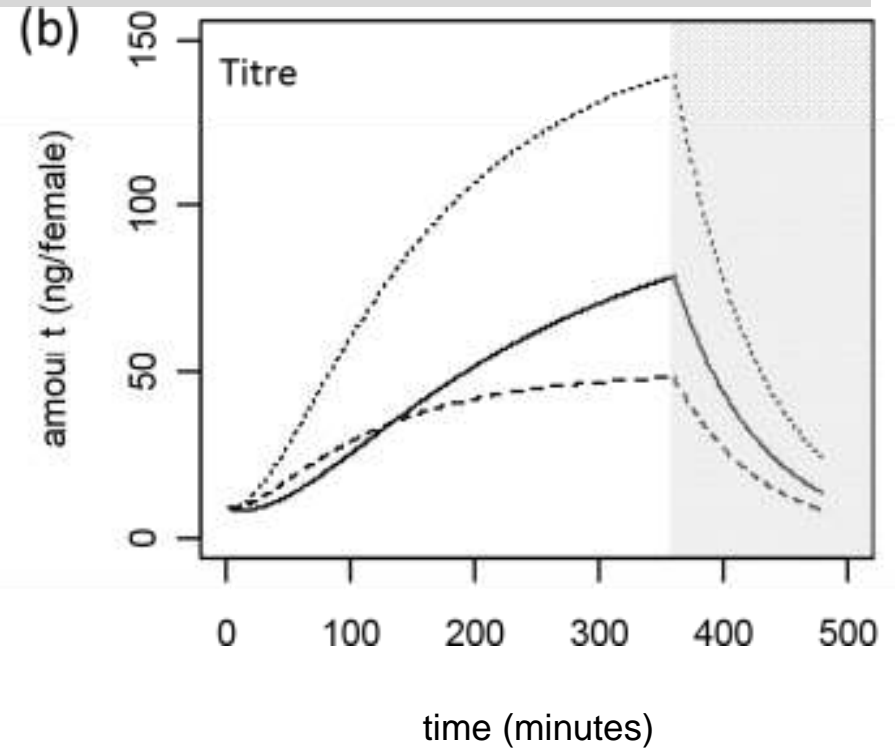

time (minutes) 
Figure 4

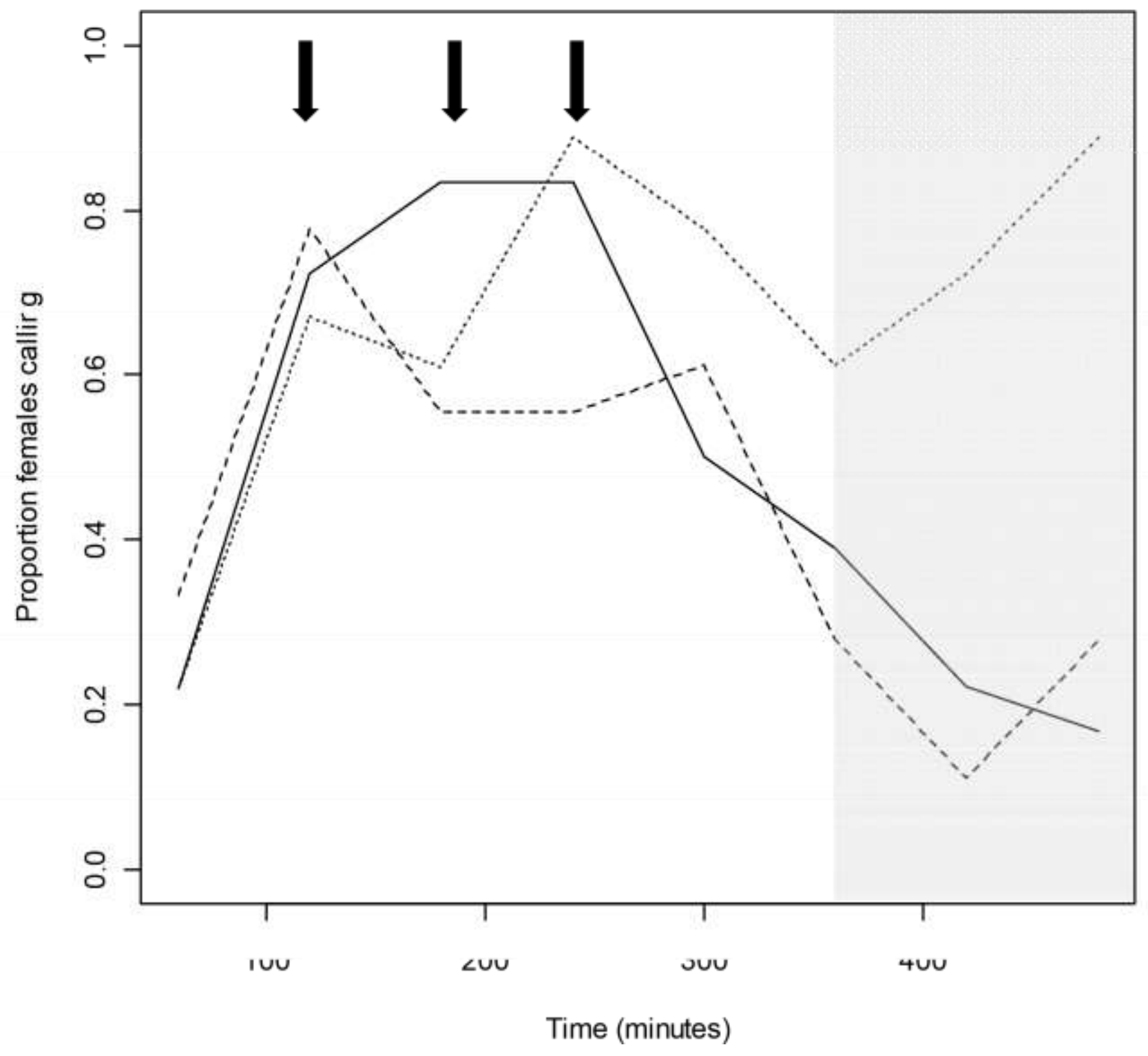



